\title{
Adult male circumcision with a circular stapler versus conventional circumcision: A prospective randomized clinical trial
}

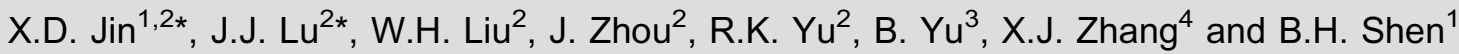 \\ ${ }^{1}$ Department of Urology, The First Affiliated Hospital, Zhejiang University School of Medicine, Hangzhou, China \\ ${ }^{2}$ Department of Urology, Beilun People's Hospital, Ningbo, China \\ ${ }^{3}$ Department of Urology, Yuyao People's Hospital, Ningbo, China \\ ${ }^{4}$ Department of Urology, Xiangshan First People's Hospital, Ningbo, China
}

\begin{abstract}
Male circumcision is the most frequently performed procedure by urologists. Safety and efficacy of the circumcision procedure requires continual improvement. In the present study, we investigated the safety and efficacy of a new male circumcision technique involving the use of a circular stapler. In total, 879 consecutive adult male patients were randomly divided into 2 groups: 441 underwent stapler circumcision, and 438 underwent conventional circumcision. The operative time, pain score, blood loss volume, healing time, treatment costs, and postoperative complications were compared between the two groups. The operative time and blood loss volume were significantly lower in the stapler group than in the conventional group $(6.8 \pm 3.1$ vs $24.2 \pm 3.2$ min and $1.8 \pm 1.8$ vs $9.4 \pm 1.5 \mathrm{~mL}$, respectively; $\mathrm{P}<0.01$ for both). The intraoperative and postoperative pain scores were significantly lower in the stapler group than in the conventional group ( $0.8 \pm 0.5$ vs $2.4 \pm 0.8$ and $4.0 \pm 0.9$ vs $5.8 \pm 1.0$, respectively; $P<0.01$ for both). Additionally, the stapler group had significantly fewer complications than the conventional group $(2.7 \%$ vs $7.8 \%$, respectively; $P<0.01)$. However, the treatment costs in the stapler group were much higher than those in the conventional group (US $\$ 356.60 \pm 8.20$ vs US $\$ 126.50 \pm 7.00$, respectively; $\mathrm{P}<0.01$ ). Most patients $(388 / 441,88.0 \%)$ who underwent stapler circumcision required removal of residual staple nails. Overall, the present study has shown that stapler circumcision is a time-efficient and safe male circumcision technique, although it requires further improvement.
\end{abstract}

Key words: Circumcision; Complication; Male; Stapler

\section{Introduction}

Male circumcision (MC) was one of the earliest operations performed by humans. This procedure has the potential to decrease the risk of sexually transmitted diseases such as human papillomavirus, genital ulcer disease, and human immunodeficiency virus (HIV) infection (1-3). Additionally, it improves penile topical hygiene and reduces the incidence of balanitis and penile cancer $(4,5)$.

Conventional $\mathrm{MC}$ as recommended by the World Health Organization (WHO) includes three techniques: the dorsal slit, the forceps-guided method, and sleeve resection (6). However, complications such as bleeding, edema, and unsatisfactory cosmetic results are still common in patients who undergo conventional MC $(7,8)$. Moreover, conventional $\mathrm{MC}$ is time consuming.

The Chinese Shang Ring was recently introduced worldwide. The use of this device is associated with a shorter operative time, lower blood loss volume, and fewer postoperative complications than in conventional $\mathrm{MC}$ $(9,10)$. However, use of the Shang Ring also has some drawbacks: more time is required for wound healing, patients must endure pain for 7 to 16 days until the ring can be removed, and wound dehiscence is relatively common after the ring is removed because the procedure is sutureless.

The circular stapler, a new disposable circumcision device, has been developed for commercial use in China (Henry Medical Device Company, Figure 1). It includes two parts: an inner bell and an outer bell. The inner bell is designed to protect the glans. The outer bell comprises a circular blade to cut the foreskin and staples to close the wound for simultaneous hemostasis. We performed a prospective randomized clinical trial involving four urological departments to investigate the safety and efficacy of MC with a circular stapler in adult male patients.

Correspondence: Bai-Hua Shen: <shen_bh123@163.com>, <kingshoulder@163.com>.

${ }^{*}$ These authors contributed equally to this study.

Received November 20, 2014. Accepted January 28, 2015. First published online March 27, 2015. 


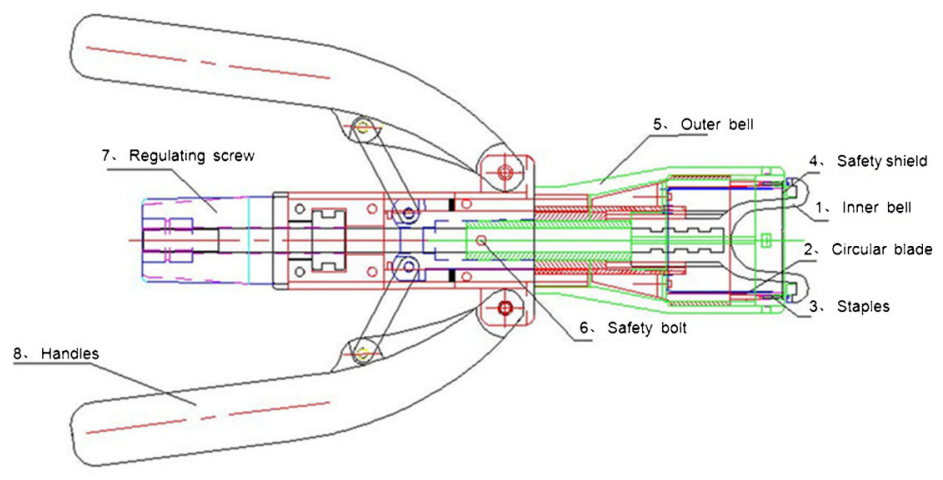

Figure 1. The circular stapler comprises an inner bell and an outer bell. The inner bell is designed to protect the glans. The outer bell has two cutting trigger handles, a regulating screw, a circular blade, staples, a safety shield, and a bolt. The circular blade and staples are hidden in the outer bell and protected by the safety shield and bolt.

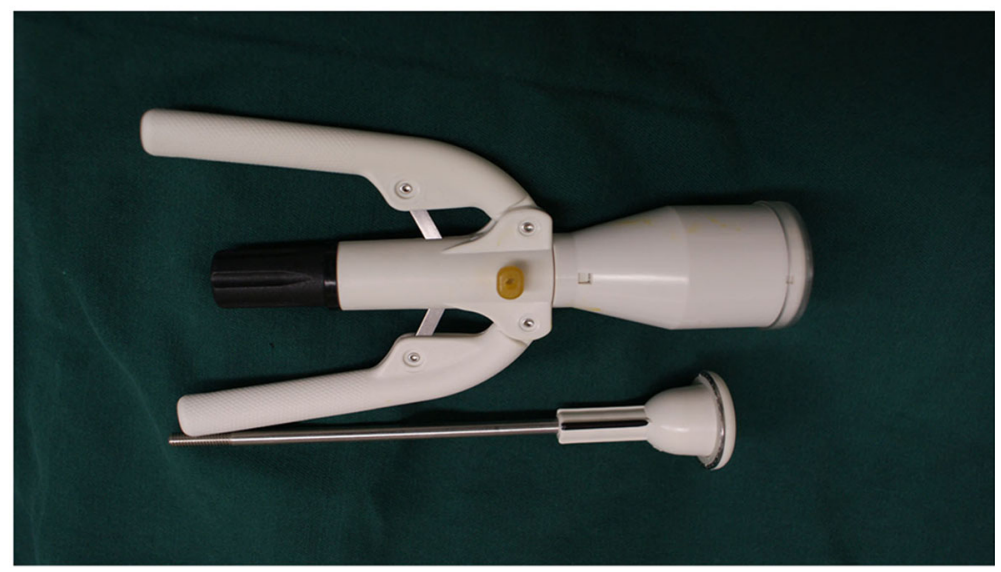

\section{Patients and Methods}

\section{Patient selection}

This clinical trial was approved by the Zhejiang University Ethics Committee, and patients provided written informed consent before undergoing the procedures. All enrolled patients were adult men 18 to 70 years of age with a redundant prepuce or phimosis. The exclusion criteria were acute infection of the genitalia (acute posthitis or balanitis), a thickened prepuce secondary to chronic inflammation, severe foreskin adhesion, or other contraindications to MC such as a concealed penis or sexually transmitted disease.

In total, 879 patients were prospectively enrolled in the present study from March 2013 to March 2014. The patients were from four urological departments (The First Affiliated Hospital, Beilun People's Hospital, Yuyao People's Hospital, and Xiangshan First People's Hospital) and divided into 2 groups: 441 underwent stapler circumcision, and 438 underwent conventional circumcision. All patients were advised to avoid sexual intercourse and masturbation for at least 4 weeks after circumcision.

\section{Surgical technique}

In the stapler group, the appropriate size of the stapler device was first determined by measuring the penis just below the glans. The penis was then surgically disinfected with povidone-iodine. A dorsal penile nerve block and a circumferential block were performed with $1 \%$ lidocaine, and the treatment was conducted according to a defined protocol (Figure 2).

In the conventional group, all patients underwent $\mathrm{MC}$ with the dorsal slit technique using an electric scalpel (Sanli Medical Equipment Company, China) in accordance with the WHO guidance manual. All circumcisions were performed by well-trained urologists.

\section{Patient follow-up and data collection}

All patients were followed up 1 day; 1, 2, and 3 weeks; and 1 and 3 months after surgery. Additionally, an investigator called each patient to inquire about the wound condition until complete healing was achieved. Fifty-six patients who underwent the stapler technique were followed up 1 year after circumcision. The following data were collected and compared between the two groups: operative time, pain score (intraoperative and $1 \mathrm{~h}$ after surgery), blood loss volume, postoperative complications, healing time, and treatment costs. We used an internationally accepted visual analog scale to evaluate pain. Mild edema was considered to be present when the perimeter of the inner foreskin layer, including the edema, was $\leq 30 \%$ of the perimeter of the penile shaft. If the perimeter was $>30 \%$ of the penile shaft, the edema was classified as severe. Wound dehiscence 

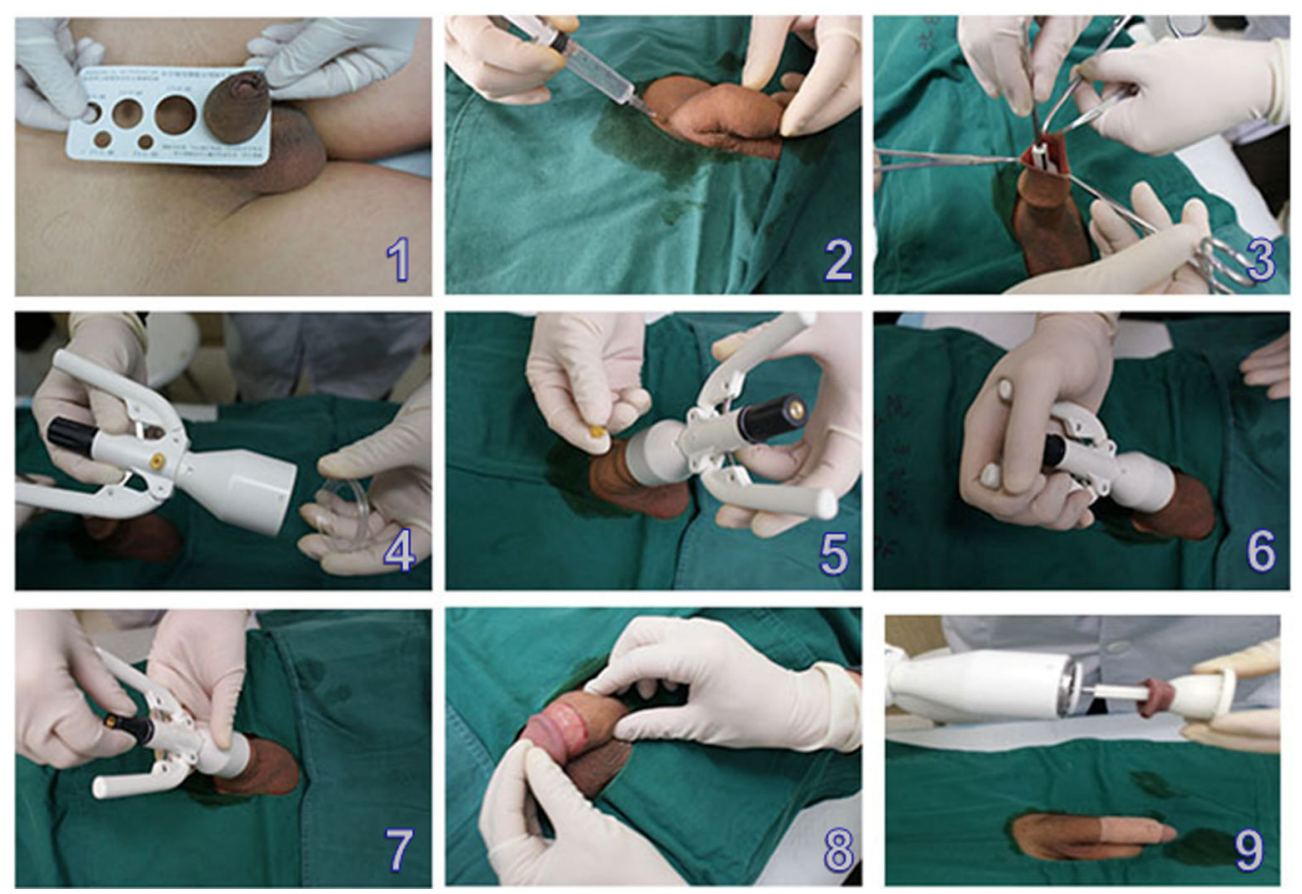

Figure 2. 1, The penis is measured just below the glans to determine the appropriate size of the stapler device. 2, After surgically scrubbing the penis with povidone-iodine, a dorsal penile nerve block and circumferential block are performed with $1 \%$ lidocaine. 3 , The inner bell is placed inside the foreskin to cover the glans; the edge of the bell is at the level of the coronal sulcus. If the patient has severe phimosis, a dorsal slit should be made to correctly position the inner bell. 4 , The safety shield is removed from the outer bell. 5 , The outer bell is placed over the inner bell. The frenulum should be kept intact. The safety bolt is then removed. 6 , The screw is rotated clockwise to sandwich the foreskin tightly; the handles are triggered to cut the foreskin and the wound is closed by staples at the same time. 7 , The device is unscrewed and removed. 8 , The wound in the foreskin is checked and pressed with gauze for 1 to 2 min to stop any bleeding. 9, Hemostasis is achieved with a compression bandage.

was defined as a $>2-\mathrm{mm}$ separation of the wound edge. Postoperative bleeding was defined as bleeding that required suture closure. The healing time was defined as the time point at which the crusts of the wound disappeared and the healing line totally appeared. All patients were asked about their satisfaction with the procedure by a questionnaire used by Yue et al. (9) 3 months after surgery.

\section{Statistical analysis}

All statistical analyses were performed with SPSS $^{\circledR}$, version 15.0 (SPSS Inc., USA). A $t$-test was used to compare the operative time, blood loss volume, healing time, and treatment costs between the two groups. We used the nonparametric Mann-Whitney test to compare pain scores and the chi-squared test to compare the incidence of complications. Statistical significance was defined as a $P$ value of $<0.05$.

\section{Results}

\section{Patient characteristics}

Patient characteristics were well balanced between the two groups. There were no statistically significant differences in age, sexual experience, or infection history.
More patients in the conventional group had phimosis. Table 1 lists the characteristics of all patients in this study.

\section{Outcomes of stapler versus conventional circumcision}

Table 2 compares the outcomes of stapler and conventional circumcision. The operative time and blood loss volume were significantly lower in the stapler group than in the conventional group $(6.8 \pm 3.1$ vs $24.2 \pm 3.2 \mathrm{~min}$

Table 1. Characteristics of patients who underwent stapler or conventional circumcision.

\begin{tabular}{lcc}
\hline & $\begin{array}{c}\text { Stapler group } \\
(\mathrm{n}=441)\end{array}$ & $\begin{array}{c}\text { Conventional } \\
\text { group }(\mathrm{n}=438)\end{array}$ \\
\hline Age (years) & $26.9 \pm 9.1$ & $25.3 \pm 7.1$ \\
Sexual experience $(\mathrm{n}, \%)$ & $220(50.0 \%)$ & $208(47.5 \%)$ \\
Infection history (n, \%) & $120(27.2 \%)$ & $131(29.9 \%)$ \\
Phimosis (n, \%) & $30(6.8 \%)^{*}$ & $49(11.2 \%)$ \\
\hline
\end{tabular}

Age is reported as means $\pm S D$. There were no significant differences between groups except in phimosis rate. ${ }^{*} \mathrm{P}<0.05$ compared to conventional group ( $t$-test and chi-squared test). 
Table 2. Comparison between stapler group and conventional group.

\begin{tabular}{lccr}
\hline Items & Stapler group $(\mathrm{n}=441)$ & Conventional group $(\mathrm{n}=438)$ & $\mathrm{P}$ \\
\hline Operative time $(\mathrm{min})$ & $6.8 \pm 3.1$ & $24.2 \pm 3.2$ & $<0.001$ \\
Blood loss $(\mathrm{mL})$ & $1.8 \pm 1.8$ & $9.4 \pm 1.5$ & $<0.001$ \\
Pain score & & & \\
$\quad$ Intraopertive & $0.8 \pm 0.5$ & $2.4 \pm 0.8$ & $<0.001$ \\
$\quad$ Postoperative & $4.0 \pm 0.9$ & $5.8 \pm 1.0$ & $<0.001$ \\
Healing time (days) & $12.5 \pm 1.8$ & $14.4 \pm 2.1$ & $<0.001$ \\
Satisfaction & $402 / 441(91.2 \%)$ & $395 / 438(90.2 \%)$ & 0.619 \\
Cost (US dollars) & $356.60 \pm 8.20$ & $126.50 \pm 7.00$ & $<0.001$ \\
Complication & $12(2.7 \%)$ & $34(7.8 \%)$ & $<0.001$ \\
$\quad$ Bleeding & 5 & 8 & 0.395 \\
Wound dehiscence & 0 & 5 & 0.030 \\
Severe edema & 5 & 18 & 0.006 \\
Infection & 2 & 3 & 0.994 \\
\hline
\end{tabular}

Data are reported as mean \pm SD or number (\%). The visual analog scale was used to evaluate pain. The Mann-Whitney, $t$ and chi-squared tests were used for statistical analyses.

and $1.8 \pm 1.8$ vs $9.4 \pm 1.5 \mathrm{~mL}$, respectively; $\mathrm{P}<0.01$ for both). The intraoperative and postoperative pain scores were significantly lower in the stapler group than in the conventional group $(0.8 \pm 0.5$ vs $2.4 \pm 0.8$ and $4.0 \pm 0.9$ vs $5.8 \pm 1.0$, respectively; $\mathrm{P}<0.01$ for both). Additionally, the stapler group had a significantly lower complication rate than did the conventional group $(2.7 \%$ vs $7.8 \%$, respectively; $\mathrm{P}<0.01)$. However, the treatment costs were much higher in the stapler group than in the conventional group $(2215.2 \pm 51.2$ Yuan (US\$356.60 \pm 8.20$)$ vs $785.5 \pm 43.5$ Yuan (US\$126.50 \pm 7.00 ), respectively; $P<0.01$ ). Similar patient satisfaction was achieved in the two groups.

Figure 3 shows the typical recovery process of stapler circumcision. There were no significant complications in either group. Most patients developed foreskin edema; mild edema usually disappeared 2 weeks after surgery, whereas severe edema lasted 1 to 3 months. Severe edema occurred in 5 of 441 patients who underwent stapler circumcision and in 18 of 438 patients who underwent conventional circumcision. In the stapler group, no patients developed wound dehiscence, but five patients developed postoperative bleeding. In the conventional group, five and eight patients developed wound dehiscence and postoperative bleeding, respectively. Wound dehiscence of $<5 \mathrm{~mm}$ was treated conservatively. If dehiscence of $>5 \mathrm{~mm}$ occurred in combination with bleeding, interrupted absorbable 5-0 sutures were applied. Infection was controlled by oral antibiotics such as levofloxacin.

A total of 42 patients $(9.5 \%)$ in the stapler group required stitches because of bleeding or incomplete wound closure during the operation. The staples usually began to fall off spontaneously 10 days after surgery. If they did not fall off completely, the residual staples had to be removed by the surgeon within 1 month after surgery.
The staples began to fall off spontaneously 10 days after surgery in $20 \%$ of the patients $(86 / 441)$; they did not fall off at all in $5 \%$ of the patients (22/441); and they fell off within 1 month in $95 \%$ of the patients (419/441). However, $88 \%$ of the patients $(388 / 441)$ still required removal of residual staples by their surgeon.

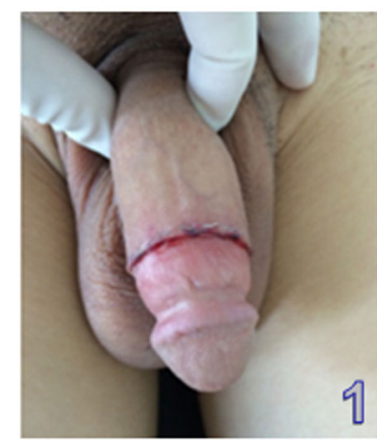

One day after surgery

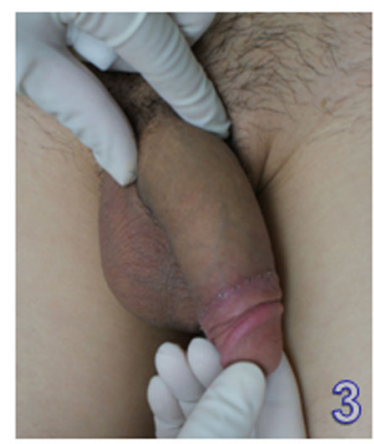

Two weeks after surgery

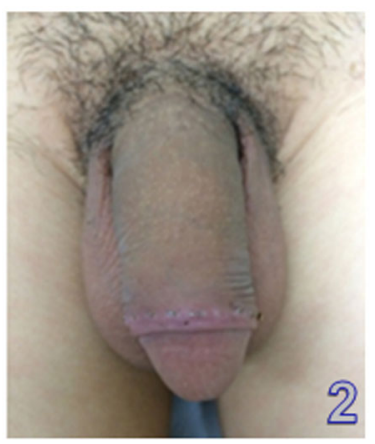

One week after surgery

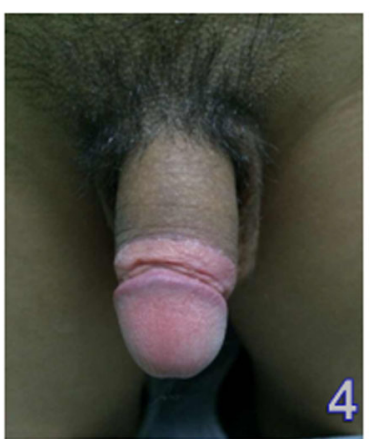

One month after surgery
Figure 3. Typical recovery process of stapler circumcision. 
Among the 879 patients who underwent either stapler or conventional circumcision, we found no penile deformities or any long-term complications such as meatal stenosis, skin bridging, or others.

\section{Discussion}

$\mathrm{MC}$ is the most frequently performed procedure by urologists. Approximately 1.4 million $\mathrm{MC}$ procedures are performed annually in US medical settings (11). The WHO recommends three conventional methods to perform circumcision, and these techniques are used worldwide. However, conventional circumcision still has some drawbacks: it requires training; it may have a high complication rate, especially in some African countries, where circumcision is often performed by poorly trained and underequipped health workers $(12,13)$; and it takes time, even when performed by experienced surgeons, which challenges the medical MC scale-up for HIV prevention in Africa (14).

The circular stapler is a new device used to perform circumcision. It was commercially developed in China and is applied in some Chinese hospitals. In line with a study by Yuan et al. (15), our data show that MC with a stapler has some advantages: a short operative time, minimal pain, and a low blood loss volume comparable with those of another new circumcision device, the Chinese Shang Ring $(9,10)$. Therefore, the stapler also has the potential to be used in high-volume settings by health care providers with minimal training and experience because of its simplicity and short operative and recovery times. Currently, its most substantial deterrent to widespread use is cost. A reusable version of the circular stapler might be a solution.

Our study has shown that stapler circumcision is associated with fewer complications than conventional circumcision, especially with respect to severe edema and wound dehiscence. These advantages of stapler circumcision may be due to the short operative time, minimal tissue injury, and lack of electrocautery. A comparison of the frequency of complications across studies has been hampered by the use of different research methods and lack of standardization. Based on the literature and our experience, we believe that the stapler still has some advantages over the Shang Ring $(10,16,17)$. First, the foreskin is cut completely with the stapler, which causes less pain after the operation. In contrast, patients who undergo circumcision with the Shang Ring experience pain for 7 to 16 days until the ring is removed. Based on patients' self-reports, this postoperative pain can be very severe when spontaneous or nocturnal erection occurs. Second, patients who undergo stapler circumcision have shorter healing time, In patients treated with the Shang Ring, removal of the foreskin is obtained by necrosis, which leads to a relatively ischemic edge. After ring removal, more time is required to reconstruct the local blood circulation for wound healing. Third, patients who undergo stapler circumcision have less wound dehiscence. Patients treated

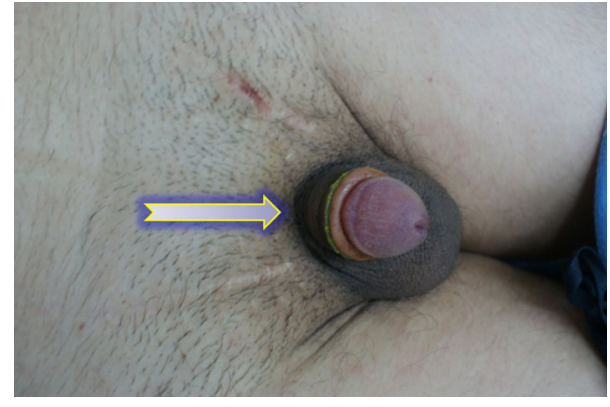

Figure 4. A plastic gasket (arrow) is present underneath the staples.

with the Shang Ring have a greater possibility of wound dehiscence because no suturing is performed around the wound. Wound dehiscence usually develops when spontaneous or nocturnal erection occurs. However, these advantages should be further tested in a well-designed head-to-head randomized clinical trial.

The main disadvantage of the stapler is the need to remove residual staples after surgery. Most patients (388/ $441,88.0 \%$ ) who underwent stapler circumcision in the present study required removal of residual staples by their surgeon. We recently added a plastic gasket underneath the staples (Figure 4) and are evaluating its impact on spontaneous staple removal. The other solution is to improve the material of the staples so that they fall off spontaneously within 1 month.

In children in some countries, circumcision is mainly performed for religious reasons $(18,19)$. One study showed that neonatal $\mathrm{MC}$ is cost-effective for disease prevention (20). The herein-described circular stapler may eventually be applied to children and infants because of its simplicity and short operative and recovery times.

The main limitations of our study are that sexual function and behavior were not evaluated and that most patients were followed up for no more than 1 year.

In conclusion, the circular stapler is an easy and userfriendly device for performing MC. It is associated with a shorter operative time, lower blood loss volume, and fewer postoperative complications than conventional circumcision. This new device may greatly facilitate and standardize circumcision procedures; thus, its popularization would be valuable. With further improvement, it could become the standard MC technique.

\section{Acknowledgments}

The authors gratefully acknowledge Professor George N. Thalmann, Department of Urology, University of Bern, Switzerland for his support in writing this manuscript. This research was supported by the Qianjiang Talent Program of Science and Technology Agency of Zhejiang Province (\#2013R10045). 


\section{References}

1. Albero G, Castellsague X, Giuliano AR, Bosch FX. Male circumcision and genital human papillomavirus: a systematic review and meta-analysis. Sex Transm Dis 2012; 39: 104-113, doi: 10.1097/OLQ.0b013e3182387abd.

2. Mehta SD, Moses S, Parker CB, Agot K, Maclean I, Bailey RC. Circumcision status and incident herpes simplex virus type 2 infection, genital ulcer disease, and HIV infection. AIDS 2012; 26: 1141-1149, doi: 10.1097/QAD.0b013e328352d116.

3. Bailey RC, Moses S, Parker CB, Agot K, Maclean I, Krieger $\mathrm{JN}$, et al. Male circumcision for HIV prevention in young men in Kisumu, Kenya: a randomised controlled trial. Lancet 2007; 369: 643-656, doi: 10.1016/S0140-6736(07)60312-2.

4. Hayashi $\mathrm{Y}$, Kohri K. Circumcision related to urinary tract infections, sexually transmitted infections, human immunodeficiency virus infections, and penile and cervical cancer. Int J Urol 2013; 20: 769-775, doi: 10.1111/iju.12154.

5. Larke NL, Thomas SL, dos Santos Silva I, Weiss HA. Male circumcision and penile cancer: a systematic review and meta-analysis. Cancer Causes Control 2011; 22: 1097-1110, doi: 10.1007/s10552-011-9785-9.

6. Hargreave T. Male circumcision: towards a World Health Organisation normative practice in resource limited settings. Asian J Androl 2010; 12: 628-638, doi: 10.1038/aja.2010.59.

7. Ceylan K, Burhan K, Yilmaz Y, Can S, Kus A, Mustafa G. Severe complications of circumcision: an analysis of 48 cases. J Pediatr Urol 2007; 3: 32-35, doi: 10.1016/j.jpurol.2006.02.009.

8. Wilcken A, Keil T, Dick B. Traditional male circumcision in eastern and southern Africa: a systematic review of prevalence and complications. Bull World Health Organ 2010; 88: 907-914, doi: 10.2471/BLT.09.072975.

9. Yue C, Ze-Jun Y, Wu KR, Su XJ, Hu JS, Ma JW, et al. A randomized clinical study of circumcision with a ring device versus conventional circumcision. J Urol 2012; 188: 18491854, doi: 10.1016/j.juro.2012.07.048.

10. Wu X, Wang Y, Zheng J, Shen W, Yan JA, Ji H, et al. A report of 918 cases of circumcision with the Shang Ring: comparison between children and adults. Urology 2013; 81: 1058-1063, doi: 10.1016/j.urology.2012.11.046.

11. El Bcheraoui C, Zhang X, Cooper CS, Rose CE, Kilmarx $\mathrm{PH}$, Chen RT. Rates of adverse events associated with male circumcision in U.S. medical settings, 2001 to 2010 . JAMA Pediatr 2014; 168: 625-634, doi: 10.1001/jamapediatrics. 2013.5414

12. $\mathrm{Kim} \mathrm{HH}$, Goldstein $\mathrm{M}$. High complication rates challenge the implementation of male circumcision for HIV prevention in Africa. Nat Clin Pract Urol 2009; 6: 64-65, doi: 10.1038/ ncpuro1279.

13. Sheldon WR, Nhemachena $\mathrm{T}$, Blanchard $\mathrm{K}$, Chipato $\mathrm{T}$, Ramjee G, Trussell J, et al. Male circumcision for HIV prevention: clinical practices and attitudes among healthcare providers in South Africa and Zimbabwe. Sex Transm Dis 2012; 39: 567-575, doi: 10.1097/OLQ.0b013e31824f9eaf.

14. Sgaier SK, Reed JB, Thomas A, Njeuhmeli E. Achieving the HIV prevention impact of voluntary medical male circumcision: lessons and challenges for managing programs. PLoS Med 2014; 11: e1001641, doi: 10.1371/journal.pmed. 1001641

15. Yuan Y, Zhang Z, Cui W, Gao B, Peng J, Xin Z, et al. Clinical investigation of a novel surgical device for circumcision. $J$ Urol 2014; 191: 1411-1415, doi: 10.1016/j.juro.2013.11.111.

16. Sokal DC, Li PS, Zulu R, Awori QD, Combes SL, Simba RO, et al. Randomized controlled trial of the shang ring versus conventional surgical techniques for adult male circumcision: safety and acceptability. J Acquir Immune Defic Syndr 2014; 65: 447-455, doi: 10.1097/QAI.0000000000000061.

17. Lv BD, Zhang SG, Zhu XW, Zhang J, Chen G, Chen MF, et al. Disposable circumcision suture device: clinical effect and patient satisfaction. Asian J Androl 2014; 16: 453-456, doi: 10.4103/1008-682X.127816.

18. Downs JA, Fuunay LD, Fuunay M, Mbago M, Mwakisole A, Peck RN, et al. 'The body we leave behind': a qualitative study of obstacles and opportunities for increasing uptake of male circumcision among Tanzanian Christians. BMJ Open 2013; 3.

19. Shah T, Raistrick J, Taylor I, Young M, Menebhi D, Stevens R. A circumcision service for religious reasons. BJU Int 1999; 83: 807-809, doi: 10.1046/j.1464-410x.1999.00000.x.

20. Morris BJ, Bailis SA, Wiswell TE. Circumcision rates in the United States: rising or falling? What effect might the new affirmative pediatric policy statement have? Mayo Clin Proc 2014; 89: 677-686, doi: 10.1016/j.mayocp.2014.01.001. 